(C)Auteurs (es). Cette œuvre, disponible à http://dx.doi.org/10.18162/fp.2021.581, est distribuée sous licence Creative Commons Attribution 4.0 International http://creativecommons.org/licences/by/4.0/deed.fr

Thomas Lecorre

Université CY Cergy-Paris (France) Imène Ghedamsi-Lecorre Université de Tunis (Tunisie)

\section{Étude exploratoire d'un changement potentiel de ressenti vis-à-vis des mathématiques de futurs professeurs des écoles en formation initiale}

\author{
Exploratory study of potential change on the feeling \\ with mathematics of pre-service elementary teachers
}

\title{
ésumé
}

La population des futurs professeurs des écoles en France est composée très majoritairement d'étudiants provenant de filières non scientifiques et leur ressenti vis-à-vis des mathématiques est souvent empreint de réticence, voire de défiance. Cet article étudie l'impact sur cette perception d'un cours de mathématique qui génère une expérience du couple (émotion, rationalité) dans le questionnement personnel et collectif des notions mathématiques en jeu.

\section{Mots-clés}

Formation initiale des professeurs d'école, ressenti, rationalité, débat scientifique, mathématiques, émotions.

\section{Abstract}

Pre-service elementary school teachers in France are mostly non-scientific students and their feelings with mathematics are often made of reluctance and mistrust.

An experimentation in the first year of mathematic courses was realised with the aim of changing positively these feelings. This article shows the impact of mobilizing student's rationality and emotions about mathematical notions.

\section{Keywords}

Pre-service elementary school teachers training, feelings, rationality, scientific debate, mathematics, emotion.

\section{Introduction}

De nombreuses études ont depuis longtemps pointé une forme d'anxiété en mathématiques à tous les niveaux de leur apprentissage chez un certain nombre d'élèves et d'étudiants (Adihou, 2011; Baruk, 1973; Baldrighi, Bellinzona et Pesci, 2011). Ce phénomène touche en particulier les professeurs d'école en formation initiale dans nombre de pays (Hannula, 2007). Cette anxiété est considérée comme un handicap majeur pour enseigner les mathématiques (Blanco, 2010). De nombreuses recherches ont été menées pour réduire cette anxiété chez de futurs professeurs d'école durant leur formation initiale (Hannula, 2007; Amato, 2004; Vinson, 2001). En France, le rapport de Ronzeau et Saint-Girons (2018) à propos de la mission des $\mathrm{ESPE}^{1}$ souligne que les trois quarts des étudiants inscrits en Master d'enseignement, d'éducation et de formation (MEEF1) proviennent de filières non scientifiques. Il précise que "[l]a plupart des candidats au Concours de recrutement des professeurs des écoles (CRPE) ont donc, en entrant à l'ESPE, une connaissance lointaine de certaines disciplines, en particulier les disciplines scientifiques, voire expriment une certaine réticence à leur égard.» (p. 23) Dans le cadre spécifique aux mathématiques, le défi énoncé par Villani et Torrossian (2018) de faire de son enseignement une des priorités nationales suppose un effort supplémentaire en ce qui concerne la formation initiale des professeurs. Développer un ressenti beaucoup plus apaisé aux mathématiques que nombre d'étudiants, futurs professeurs d'école, nont pas préalablement expérimenté (Smith, 2010) constitue donc un défi majeur d'une telle formation. Nous étudions dans cet article l'impact d'une expérimentation menée auprès d'étudiants de MEEF1 de l'INSPE ${ }^{2}$ de l'académie de Versailles qui vise l'amélioration de leur ressenti envers les mathématiques. Cette expérimentation repose sur une proposition pédagogique fondée sur l'expérience rationnelle et émotionnelle d'une construction collective. 


\section{Les mathématiques en formation initiale : enjeux et perspectives}

En France, les réformes successives de la formation initiale des professeurs des écoles tentent chacune de répondre à des enjeux éducatifs nationaux forts, en particulier améliorer la qualité des apprentissages ${ }^{3}$ et réduire les inégalités scolaires ${ }^{4}$. Ces différents enjeux prennent une dimension toute particulière en mathématiques où les besoins sont les plus sensibles 5 . Le rapport Villani et Torossian (2018) place sa priorité précisément au premier degré et préconise une formation initiale adaptée aux futurs professeurs d'école du fait que «80\% des impétrants sont issus des flières relevant des humanités en licence» (p. 43), autrement dit de filières non scientifiques.

Cette priorité actuelle de l'éducation nationale relève d'un constat qui lui n'est pas neuf. Peix et Tisseron (1997) avaient souligné que :

Une courte enquête réalisée auprès de formateurs de professeurs d'école à l'IUFM, fait apparaître que le niveau en mathématiques de la plupart des étudiants est souvent jugé peu satisfaisant. Plus que les performances, l'idée que les étudiants se font des mathématiques préoccupe les formateurs. Ceux-ci disent avoir couramment observé chez les étudiants de première année un sentiment d'échec ancré depuis parfois longtemps, ou bien la conviction que faire des mathématiques est une activité qui se résume soit à choisir, puis appliquer la bonne formule, soit à effectuer des raisonnements de type déductif, vus comme des exercices de pure forme, dénués de signification, et par là avant tout contraignants (Peix et Tisseron, p. 6).

À l'instar de cette citation, au-delà du niveau critique de connaissances en mathématiques des étudiants futurs professeurs d'école (Southwell, 2005 ; Newton 2012), la littérature internationale a mis en évidence au moins trois déterminants de l'impact d'une formation en mathématiques des futurs professeurs d'école : leur représentation de ce qu'est l'activité mathématique en général, leur représentation de l'apprentissage des mathématiques, et enfin leur expérience des mathématiques en milieu scolaire (Arsenault, 2010; Liljedahl et al., 2019). Ces déterminants, souvent corrélés, ont constitué le point d'appui de divers types d'interventions qui ont pour objet la modification d'une (ou de plusieurs) de ces représentations en vue d'une optimisation de leur formation. Par exemple, il existe des «approches thérapeutiques » qui visent d'abord l'anxiété à travers des dispositifs essentiellement réflexifs où l'objet est d'exprimer cette anxiété et ses conditions d'apparition en mathématiques (Hannula, 2007; Olson \& Stoehr,2019).Il existe également des dispositifs visant à procurer aux étudiants une expérience nouvelle de l'apprentissage des mathématiques susceptible de modifier leur appréhension de celles-ci, en leur faisant, par exemple, expérimenter le bonheur de la découverte en mathématique (Liljedahl, 2005). Des alternatives de formation de futurs professeurs des écoles, fondées sur le savoir mathématique, visant à améliorer leurs représentations négatives des mathématiques sont également décrites dans Liljedahl et al. (2019). Notre démarche s'inscrit dans cette dernière option, c'est-à-dire dans le choix de l'activité mathématique comme principal facteur de changement du ressenti envers les mathématiques lorsque, lors de la pratique de cette activité, la stimulation de l'émotion est une condition sine qua none au développement rationnel. 
La conjecture que nous cherchons à étudier est, par conséquent, la suivante : quand la pratique de l'activité mathématique mobilise effectivement la rationalité personnelle, alors l'expérience du couple (rationalité, émotion) qui accompagne cette pratique est de nature à modifier le ressenti des étudiants futurs professeurs des écoles envers leurs représentations des mathématiques en tant que science, discipline scolaire et objet d'apprentissage.

\section{Éléments didactiques pour l'expérience du couple (rationalité, émotion)}

\section{Rationalités et émotions}

L'étude de l'exercice de la rationalité repose ici sur le modèle des rationalités épistémiques (Lecorre, 2016). La rationalité est alors appréhendée comme "[...] un système de production et de contrôle de connaissances qui a pour vocation d'assurer la non-contradiction de ces connaissances entre elles ou avec un domaine de réalité.» (Lecorre, 2016, p. 152). Ce modèle repose sur trois types de vérités dont découlent trois rationalités : la vérité pragmatique, c'est vrai parce que ça marche, qui produit la rationalité pragmatique, la vérité empirique, c'est vrai, car ça a été reproduit de nombreuse fois, qui produit la rationalité empirique, et enfin la vérité apodictique, c'est vrai parce que cela découle d'un système d'axiomes, qui produit la rationalité théorique. Chacune de ces rationalités tente d'assurer la noncontradiction à travers trois composantes de l'activité mathématique : le mode d'existence des objets, la logique, et le mode validation. Par exemple, pour la rationalité théorique, le mode d'existence des objets est la définition, tandis que pour la rationalité pragmatique, ce mode d'existence est matériel : cet objet existe, car je peux l'exhiber, le dessiner, le toucher... Lors de la pratique de l'activité mathématique, ce modèle permet de suivre les différentes rationalités à l'œuvre, autrement dit l'émergence du sens, en particulier à travers les différentes composantes mobilisées. Les allers et retours entre rationalités sont dynamisés par des conflits de rationalités qui correspondent à des conflits cognitifs étudiés sous l'angle des rationalités. L'émergence de ces conflits de rationalités ainsi que leur résolution, ou non, s'accompagne d'un changement sur le plan des états émotionnels qui se traduit par un sentiment de surprise, d'agacement, de doute, d'impatience, de soulagement, d'émerveillement, de certitude, et bien d'autres (Damasio, 2005).

\section{Débat scientifique et émotions}

La stratégie pédagogique retenue pour l'expérimentation est celle d'un cours interactif mobilisant le «débat scientifique en classe » développé par Legrand (1993) à l'Institut de Recherche surl'Enseignement des Mathématiques (IREM) de Grenoble et portant sur le questionnement des notions fondamentales par des débats collectifs entre étudiants, débats animés par le professeur.

Concrètement, des conjectures à résoudre, soit proposées par le professeur, soit par les étudiants, sont mises au débat. Par exemple, pour la première séance, une conjecture ayant pour double objectif, soit la modélisation mathématique et la logique, a été proposée par le professeur : «Plus la clôture d'un champ de patates est longue, plus on produit de patates sur ce champ ». Lors de l'étude cette conjecture, après de nombreuses controverses, il s'avère que les étudiants conviennent que la conjecture n'est pas résoluble telle quelle (trop de facteurs en jeu : le type de patates, la météo...). Ils proposent alors, par 
un acte de modélisation, une nouvelle conjecture : «Plus le périmètre d'une figure est grand, plus son aire est grande». Létude de cette conjecture démontre que cela dépend des figures. La spécificité de la logique mathématique est convoquée pour conclure qu'un seul contre-exemple suffit pour invalider une conjecture.

De tels débats sont très animés, car les positions des étudiants sont contradictoires et reflètent la variété des rationalités en jeu. De nombreux conflits de rationalités voient le jour et conduisent les étudiants à l'expression d'émotions fortes: des tensions (mais pourquoi ne pensent-ils pas comme moi?), de l'agacement (fait-il exprès de continuer de penser cela?), de doute (et s'il avait raison de penser ainsi ?), d'assurance (je ne suis pas le seul à penser ainsi), de jubilation collective (le professeur reprend à son compte que la qualité de l'agriculteur joue un rôle dans la production de patates), de la surprise (comment peut-il penser ainsi ?), de l'émerveillement (tout devient enfin clair pour moi ou bien j'avais bien raison de penser cela), d'apaisement (quand le conflit de rationalité s'évanouit).

Lors de ces débats, ces émotions (et d'autres) jouent évidemment un rôle important dans la progression et le développement des apprentissages en lien avec les notions mathématiques en jeu (Pekrun, 2014). Mais c'est à un autre niveau que le changement de ressenti s'opère. Il s'inscrit dans une autre durée, à travers des apprentissages métamathématiques sur le long terme. La manière d'aborder un problème change, elle est accompagnée de beaucoup de patience et d'opiniâtreté. La nature même du doute se modifie, il perd sa seule signification d'ignorance et gagne celle de méthode d'investigation. Les formules et théorèmes n'apparaissent plus comme seulement à appliquer dans des cas standards, mais comme des outils très utiles pour dénouer les situations sans eux insolubles. Cette transformation progressive du sujet épistémique (rationnel), cette nouvelle façon du sujet de connaître en mathématique, effets attendus de l'expérience personnelle du couple (rationalité, émotion), sont de nature à constituer les ferments d'un changement positif de ressenti envers les mathématiques.

\section{Hypothèse centrale de transformation du ressenti}

Nous fondons les mécanismes de transformation du ressenti sur un postulat psychocognitif : lorsqu'un individu expérimente l'efficacité de sa propre rationalité, il en tire une satisfaction profonde. En effet, lorsqu'un individu s'aperçoit que l'exercice de sa propre rationalité, après une période de tâtonnement, lui permet de bien mieux appréhender une certaine réalité, il prend conscience, avec plaisir, de sa propre capacité à se saisir de la complexité du monde. Puisqu'elles reposent sur la révélation de la jouissance de la pensée propre et de sa puissance, cette satisfaction et cette prise de conscience, quand elles surviennent, sont de nature à modifier le ressenti envers les mathématiques à travers un changement de leurs représentations de l'activité mathématique, de l'apprentissage des mathématiques et des mathématiques en tant que discipline scolaire (Liljedahl, 2005). L'activité mathématique et l'apprentissage des mathématiques, entre autres, peuvent apparaître ainsi beaucoup plus clairement associés à la mobilisation de la rationalité propre et beaucoup moins à l'utilisation de solutions types et de recettes (Brousseau, 1998; Lecorre, 2018; Liljedahl et al., 2019). 


\section{Méthodologie de la recherche}

\section{Contexte de l'expérimentation}

L'expérimentation concerne les étudiants de première année de MEEF1, année de préparation du CRPE sur un site de l'ESPE de l'académie de Versailles. En général, la formation mathématique à ce niveau du cursus a essentiellement pour objet d'agir sur le niveau de connaissances de la discipline et de sa didactique, conformément au programme et aux épreuves de mathématiques du CRPE. Ici, l'expérimentation complète cet objectif par un autre : celui de modifier positivement le ressenti des étudiants envers les mathématiques.

La formation en mathématique durant la première année du master MEEF1 se déroule, conformément à la maquette de formation, essentiellement selon deux modalités : d'une part, des travaux dirigés (TD) pour des groupes de 20 à 30 étudiants et d'autre part, un cours magistral (CM) pour tous les groupes, soit un effectif d'environ 80 étudiants. Le constat de départ montre que les enseignants, sur tous les sites de l'académie, considèrent globalement le cours magistral comme inefficace au vu de la désertification progressive de l'amphithéâtre au cours de l'année et du désintérêt manifeste des étudiants, l'assiduité aux cours magistraux n'étant pas considérée par les étudiants comme obligatoire, contrairement aux travaux dirigés. C'est ce cours magistral qui est choisi pour l'expérimentation.

Le cours magistral a comporté dix séances (de 1 h 30 à 2 h chacune) et a porté sur les aspects suivants : modélisation du langage et des notions mathématiques, changement de base de numération, transition arithmétique/algèbre,géométrie euclidienne, figures homologues, dialectique grandeur/nombre, passage de la géométrie de dimension 2 à la géométrie de dimension 3, pensée naturelle de la proportionnalité. Ce cours garde le nom de cours magistral seulement pour des raisons de conformité administrative. Cependant, il n'a rien d'un cours magistral, ni sur le fond ni sur la forme (cf II.2). Néanmoins, il continue de réunir tous les étudiants du même niveau dans un amphi. Ce cours magistral est ensuite suivi de travaux dirigés (TD) de 4 h qui ont lieu quasiment chaque semaine.

\section{Modalités d'étude de l'expérimentation}

Deux niveaux d'études ont été envisagés. D'abord, un niveau local où l'on étudie les situations du cours magistral selon le modèle des ingénieries didactiques avec toutes les étapes que cela comporte, conception, analyse a priori, analyse a posteriori (Artigue, 1990). Ce niveau de l'étude ne fera pas l'objet de cet article. Ensuite, un niveau global, à l'échelle de l'année, d'étude des effets du dispositif sur

le ressenti en comparant des états initiaux et finaux des étudiants à l'aide d'un même questionnaire administré en amont et en aval de l'expérimentation.

\section{Le questionnaire a priori et a posteriori}

Nous explorerons les ressentis des étudiants sur trois dimensions de perception des mathématiques : la science, la discipline scolaire et leur apprentissage, et étudierons l'éventuel bougé vis-à-vis de ces trois dimensions. 
Le même questionnaire anonyme est donc donné en début d'année puis en fin d'année afin de comparer les évolutions du ressenti des élèves. Ce questionnaire comporte deux parties. Une première partie permet de mettre en évidence les variables d'identification de l'étudiant et de son parcours scolaire et universitaire (âge, sexe, type de bac, type de licence, etc.). Une deuxième partie comporte neuf questions sous forme d'affirmations à classer selon une échelle de Likert à quatre degrés (très en accord, assez d'accord, assez en désaccord, très en désaccord). Ce questionnaire reprend les questions de l'enquête internationale TIMMS 2015 (grade 8, mathematics, item 20). Cette enquête, testée et adaptée 6 fois ces 20 dernières années, est destinée à éclairer les politiques publiques en matière d'éducation à la science. Elle permet, en particulier, d'étudier à la fois le niveau des élèves, mais aussi leur représentation des mathématiques. C'est cette dernière partie que nous avons retenue, car elle correspond à notre problématique sur le ressenti vis-à-vis des mathématiques. Ces neuf questions peuvent se regrouper en trois groupes de trois questions, chaque groupe testant une seule des trois variables : les mathématiques comme discipline scolaire, les mathématiques comme science, l'apprentissage des mathématiques.

La variable «les mathématiques comme discipline scolaire» comporte trois affirmations (entre parenthèses leur numéro d'ordre donné dans le questionnaire) : J'aurais aimé ne pas étudier les mathématiques (b); Les mathématiques sont une de mes matières préférées (i); Je suis impatient d'aller en cours de mathématiques (h).

La variable «les mathématiques comme science» comporte trois affirmations: Les mathématiques sont ennuyeuses (c); J'aime les mathématiques (e); J'aime résoudre des problèmes mathématiques (g).

La variable sur «l'apprentissage des mathématiques» comporte trois affirmations : J'aime apprendre les mathématiques (a); J'apprends beaucoup de choses intéressantes en mathématiques (d); J'aime n'importe quel travail scolaire incluant des nombres (f).

Les questions posées, pour chacune des variables, devraient nous permettre de quantifier les réponses des étudiants et d'être en mesure d'évaluer leurs ressentis par rapport à chacune de ces variables.

\section{Modalités de recueil et de traitement des données}

Le recueil des questionnaires est réalisé en début d'année et en fin d'année. Le recueil des 80 étudiants n'a pas été effectué en entier pour diverses raisons techniques et seuls 50 questionnaires sont finalement étudiés.

Pour les besoins de l'étude statistique préliminaire de cohérence des réponses à l'intérieur de chaque variable, nous avons codé les réponses de 1 à 4 après les avoir ordonnées dans un sens croissant, qui va de «très en accord» jusqu'à "très en désaccord», ou décroissant en fonction de la question posée. Par exemple, pour la variable «les mathématiques comme discipline scolaire», seules les affirmations «J'aurai aimé ne pas étudier les mathématiques» et "Les mathématiques sont ennuyeuses» ont été ordonnées dans le sens décroissant. Les résultats de l'analyse des correspondances (sur SPSS) font apparaître une bonne corrélation des réponses pour chaque variable; ainsi, les choix de regroupements des questions par trois ne sont pas invalidés par cette étude sur le plan de la cohérence des réponses. 
Afin de pouvoir identifier les bougés de ressenti pour chaque variable, nous avons catégorisé les réponses pour chaque variable selon trois groupes ( $\mathrm{A}$ très positif, $\mathrm{B}$ mitigé, $\mathrm{C}$ très négatif). Pour chaque variable, on compte le nombre de réponses positives dans le sens des ressentis $(1=$ «très en accord», $2=$ «assez d'accord»). Le nombre de réponses positives peut être égal à 3 , si toutes les réponses des trois questions correspondantes à cette variable sont positives; ou bien égal à 2 , si deux réponses sont positives; ou encore 1 , si seule une réponse est positive et enfin 0 si aucune des trois réponses n'est positive.

À partir de ce nombre de réponses, on compose, pour chaque variable, trois groupes (A, B et $\mathrm{C})$ :

- Le groupe A, pour une variable donnée, est composé des individus ayant un nombre de réponses positives pour la variable en question égal à 3 ou à 2 . Les individus du groupe $A$, pour cette variable, ont un ressenti plutôt (très) positif.

- Le groupe B, pour une variable donnée, est composé des individus ayant un nombre de réponses positives égal à 1 pour la variable en question. Les individus du groupe $\mathrm{B}$, pour cette variable, ont un ressenti plutôt mitigé (avec une coloration plutôt négative).

- Le groupe C, pour une variable donnée, est composé des individus ayant un nombre de réponses positives égal à 0 pour la variable en question. Les individus du groupe $\mathrm{C}$, pour cette variable, ont un ressenti très négatif.

\section{Résultats du questionnaire en amont de la formation}

\section{Profils des étudiants de MEEF1 en première année de la promotion 2018-2019}

Sur 50 étudiants, 3 ont plus de 40 ans, 7 ont entre 30 et 40 ans et 40 ont entre 20 et 30 ans, pour une moyenne globale de 25 ans et demi. Il y a 42 femmes et 8 hommes. La répartition des filières de bac est la suivante : S : $26 \% \mathrm{~L}: 30 \% \mathrm{ES}: 30 \%$ Autre : $14 \%$. Lorigine des licences se répartie ainsi : Science $42 \%$, Littéraire $32 \%$, Gestion $10 \%$, Droit $6 \%$ et Autres $10 \%$. Enfin, la grande majorité des étudiants proviennent de la région parisienne.

\section{Résultats sur le ressenti vis-à-vis des mathématiques}

Les résultats initiaux (tableau 1) pour les trois variables se présentent par répartition dans ces trois groupes ainsi :

\section{Tableau 1}

Répartition initiale des variables dans les groupes $A, B$ et $C$

\begin{tabular}{cccc}
\hline & Discipline & Apprentissage & Science \\
\hline A & $42 \%$ & $64 \%$ & $56 \%$ \\
\hline B & $38 \%$ & $16 \%$ & $22 \%$ \\
\hline C & $20 \%$ & $20 \%$ & $22 \%$ \\
\hline
\end{tabular}


Comme on peut le constater, pour chaque variable, seuls $20 \%$ des étudiants sont finalement concernés par un ressenti très négatif (groupe $\mathrm{C}$ ). Un nombre important d'étudiants se trouve plutôt (très) positif dans son ressenti vis-à-vis des mathématiques indépendamment de la variable (groupe A). Cependant, un nombre conséquent d'étudiants se trouve aussi dans des groupes à ressentis plutôt négatifs vis-à-vis des mathématiques (groupe B et C) : $36 \%$ des étudiants pour la variable «Apprentissage», $44 \%$ pour la variable «Science» et $58 \%$ pour la variable «Discipline». Ainsi, si cette répartition quantitative est plus positive et nuancée que la littérature ne le laissait présager, cela s'explique sans doute par la surreprésentation des étudiants issus de filières scientifiques (42\%) par rapport à la moyenne nationale (25\%) en MEEF1. Il subsiste néanmoins, dans ce groupe de 50 étudiants, un potentiel substantiel d'amélioration à propos des objectifs. D'autre part, pour les autres étudiants, ceux du groupe A, le cours de mathématiques prévu par l'expérimentation est de nature à répondre à des besoins de connaissances. On remarquera enfin que la variable discipline se détache nettement des deux autres variables, sa répartition étant beaucoup plus importante dans les groupes $\mathrm{B}$ et $\mathrm{C}$ comparée aux deux autres variables.

\section{L'aval de la formation : discussion des résultats}

\section{Étude quantitative : résultats des questionnaires}

Pour les résultats du questionnaire après expérimentation, nous notons A', B' et C' les groupes formés conformément aux formules utilisées respectivement pour former les groupes $\mathrm{A}, \mathrm{B}$ et $\mathrm{C}$ relatifs au questionnaire avant expérimentation. Les 50 réponses finales sont réparties ainsi selon les trois variables et les trois groupes :

\section{Tableau 2}

Répartition finale des variables dans les groupes $A$ ', $B$ ' et $C$ '

\begin{tabular}{cccc}
\hline & Discipline & Apprentissage & Science \\
\hline $\mathrm{A}^{\prime}$ & $46 \%$ & $72 \%$ & $68 \%$ \\
\hline $\mathrm{B}^{\prime}$ & $34 \%$ & $12 \%$ & $16 \%$ \\
\hline $\mathrm{C}^{\prime}$ & $20 \%$ & $16 \%$ & $16 \%$ \\
\hline
\end{tabular}

On peut remarquer que la nouvelle répartition est plus favorable au groupe A' qu'aux groupes B' et C'; cependant, si cela indique globalement une amélioration du ressenti, celle-ci reste mesurée. 
La répartition des mouvements des individus d'un groupe à l'autre pour chaque variable est synthétisée dans le tableau suivant :

Tableau

Répartition des mouvements d'un groupe à l'autre pour chaque variable

\begin{tabular}{cccccccccc}
\hline & \multicolumn{3}{c}{ Discipline } & \multicolumn{4}{c}{ Apprentissage } & \multicolumn{3}{c}{ Science } \\
\hline & $\mathrm{A}^{\prime}$ & $\mathrm{B}^{\prime}$ & $\mathrm{C}^{\prime}$ & $\mathrm{A}^{\prime}$ & $\mathrm{B}^{\prime}$ & $\mathrm{C}^{\prime}$ & $\mathrm{A}^{\prime}$ & $\mathrm{B}^{\prime}$ & $\mathrm{C}^{\prime}$ \\
\hline $\mathrm{A}$ & $34 \%$ & $6 \%$ & $2 \%$ & $54 \%$ & $6 \%$ & $4 \%$ & $48 \%$ & $6 \%$ & $2 \%$ \\
\cline { 2 - 10 } $\mathrm{B}$ & $8 \%$ & $22 \%$ & $8 \%$ & $8 \%$ & $4 \%$ & $4 \%$ & $12 \%$ & $8 \%$ & $2 \%$ \\
\hline $\mathrm{C}$ & $4 \%$ & $6 \%$ & $10 \%$ & $10 \%$ & $2 \%$ & $8 \%$ & $8 \%$ & $2 \%$ & $12 \%$ \\
\hline
\end{tabular}

Pour la variable «Discipline», 8 \% de l'ensemble des individus sont passés du groupe A au groupe B' ou C', $8 \%$ de l'ensemble des individus sont passés du groupe B au groupe C', soit $16 \%$ des étudiants ayant pour la variable «Discipline» un changement de ressenti négatif. Pour la même variable, $18 \%$ ont un changement de ressenti positif.

Plusieurs constats s'imposent :

1) Une certaine stabilité des groupes : pour chacune des variables, les changements de groupe ne concernent environ qu'un seul tiers de l'ensemble des individus.

2) Les changements très significatifs (entre $A$ et $C$ ) sont rares du groupe $A$ au groupe $C$ '(moins de $4 \%$ de la totalité des étudiants pour chaque variable) et moins rares du groupe $\mathrm{C}$ au groupe $\mathrm{A}^{\prime}$ ( $4 \%$ pour «Discipline», $10 \%$ pour «Apprentissage» et $8 \%$ pour «Science»). Ce constat est plus clair lorsqu'on rapporte ces changements à la quantité d'étudiants concernés par la potentialité de ces changements (ceux du groupe $\mathrm{A}$ pour le passage de $\mathrm{A}$ à C'et ceux du groupe $\mathrm{C}$ pour le passage de $\mathrm{C}$ à $\mathrm{A}^{\prime}$ ). Ainsi, ces changements très négatifs (de $\mathrm{A}$ à $\mathrm{C}^{\prime}$ ) concernent $5 \%$ des étudiants du groupe A pour la variable «Discipline», $7 \%$ pour la variable «Apprentissage », et $4 \%$ pour la variable «Science». Ces changements très négatifs concernent essentiellement des étudiants dans la position inférieure du groupe $\mathrm{A}$ (i.e. ayant seulement deux réponses positives de type 2 "plutôt d'accord») à une position supérieure du groupe $\mathrm{C}$ (ayant surtout des réponses négatives de types 3 "plutôt pas d'accord»). Ces changements très négatifs et très minoritaires illustrent le type de loi normale qui régit l'essentiel des comportements humains.

Les changements très positifs (de $\mathrm{C}$ à $\mathrm{A}^{\prime}$ ) sont alors beaucoup plus importants. Ils concernent $20 \%$ de ceux qui sont dans le groupe $\mathrm{C}$ pour la variable «Discipline», $50 \%$ pour la variable «Apprentissage» et $36 \%$ pour la variable «Science». On peut conclure que des changements très positifs et quantitativement très significatifs (du groupe $\mathrm{C}$ au groupe $\mathrm{A}^{\prime}$ ) ont été opérés par les étudiants assez massivement pour chaque variable (un peu moins pour la variable «Discipline»), tandis que beaucoup moins de changements opposés ont vu le jour. 
3) De manière générale, quand on relativise les pourcentages de mouvements aux individus concernés par la possibilité de ces mouvements, on peut constater que les mouvements négatifs (de A à $\mathrm{B}$ ou $\mathrm{C}$ et de $\mathrm{B}$ à $\mathrm{C}$ ) sont bien inférieurs aux mouvements positifs (de $\mathrm{C}$ à $\mathrm{A}$ ou $\mathrm{B}$ et de $\mathrm{B}$ à $\mathrm{A}$ ). Par exemple, pour la variable «Discipline», on peut remarquer que les mouvements positifs concernent $18 \%$ (de la population totale) pour une population susceptible d'effectuer ce mouvement positif de $58 \%$ (les groupes $\mathrm{B}$ et $\mathrm{C}$ par rapport à la population totale), soit un mouvement positif relatif de $31 \%$ de ceux qui étaient en position de pouvoir faire ce mouvement. Les autres mouvements relatifs sont récapitulés dans le tableau 4 et montrent, pour chaque variable, que le mouvement relatif positif est systématiquement largement supérieur au mouvement relatif négatif.

\section{Tableau 4}

Pourcentages de changements relatifs à la population pouvant effectuer ces mouvements

\begin{tabular}{cccc}
\hline & Discipline & Apprentissage & Science \\
\hline $\begin{array}{c}\text { Changements } \\
\text { positifs }\end{array}$ & $31 \%$ & $56 \%$ & $50 \%$ \\
\hline $\begin{array}{c}\text { Changements } \\
\text { négatifs }\end{array}$ & $20 \%$ & $18 \%$ & $13 \%$ \\
\hline
\end{tabular}

4) D'après le tableau 4, les changements positifs sont beaucoup plus sensibles pour les variables «Apprentissage» et «Science» que pour la variable «Discipline».

À partir de ces constats, on peut dire qu'une amélioration du ressenti vis-à-vis des mathématiques a eu lieu de manière importante. Mais on ne peut imputer qu'une partie de cette transformation au cours magistral. En effet, les travaux dirigés représentent un volume horaire beaucoup plus significatif que le seul cours magistral. Cela peut expliquer, par exemple, que la variable «Discipline» soit celle pour laquelle les changements réalisés sont les plus faibles : le cours magistral, tel qu'il est conçu, vise beaucoup plus les deux autres variables et la variable «Discipline scolaire» est davantage travaillée dans les TD. À ce sujet, sur les quatre groupes de TD (I, II, III et IV) (36 étudiants), seul le groupe de TD II (14 étudiants) a eu un enseignement qui prolongeait en TD explicitement les pratiques du cours magistral (même intervenant et même pratique). Et lorsqu'on compare les résultats du questionnaire séparément (groupe de TD II, et réunion des groupes de TD I, III et IV), le contraste est clair (tableau 5 et 6$)$.

Tableau 5

\begin{tabular}{cccc}
\hline & Discipline & Apprentissage & Science \\
\hline Mouvements positifs & $25 \%$ & $50 \%$ & $53 \%$ \\
\hline Mouvements négatifs & $27 \%$ & $23 \%$ & $18 \%$ \\
\hline
\end{tabular}

Pourcentage des différents mouvements, relativement aux individus pouvant réaliser ces mouvements, par variable pour les groupes de TD I, III et IV 
Tableau 6

\begin{tabular}{cccc}
\hline & Discipline & Apprentissage & Science \\
\hline Mouvements positifs & $44 \%$ & $100 \%$ & $40 \%$ \\
\hline Mouvements négatifs & $7 \%$ & $7 \%$ & $0 \%$ \\
\hline
\end{tabular}

Pourcentage des différents mouvements, relativement aux individus pouvant réaliser ces mouvements, par variable pour le groupe II

La significativité des tableaux précédents est obtenue par un test de chi2 de Pearson (logiciel R) et donne un seuil de rejet de $\mathrm{H}_{0}$ (non-indépendance des variables groupes et type de mouvement) avec une valeur de $\mathrm{p}$ inférieure à 0,01 pour les trois variables, soit un rejet de $\mathrm{H}_{0}$ avec une probabilité de $99 \%$. Ainsi, la comparaison de ces résultats montre que les mouvements vers un ressenti positif sont globalement plus importants dans le groupe de TD II que dans les autres groupes, mais surtout que les mouvements vers un ressenti plus négatif sont très inférieurs dans ce groupe de TD II. Cela nuance évidemment l'impact du cours magistral, puisqu'une partie des constats précédents se fondent sur l'ensemble des étudiants où le groupe de TD II, bien que représentant seulement le quart de l'ensemble des étudiants, joue un rôle significatif sur les résultats globaux, manifestement du seul fait d'avoir un TD différent. Mais cela confirme également que la proposition pédagogique prévue et réalisée, lorsquelle poursuit, en TD, le même objectif que celui assigné au cours magistral et avec la même méthode, a encore plus d'impact vis-à-vis de cet objectif. Ce constat est de nature à accréditer les effets de cette proposition pédagogique sur le ressenti des étudiants vis-à-vis des mathématiques.

À partir de cette comparaison, on peut également remarquer que les variables "Apprentissage» et «Science», celles qui sont essentiellement travaillées en cours magistral, ont les plus forts taux de mouvements positifs dans les deux groupes de TD. Concernant la variable «Discipline», celle davantage travaillée en TD, le taux de mouvement positif est bien moindre que pour les deux autres variables dans les groupes de TD I, III et IV, alors que ce constat n'est pas aussi marqué pour le groupe de TD II. Ceci tend à confirmer l'impact du cours magistral sur les variables qui y sont essentiellement travaillées et celui du TD sur la variable «Discipline» lorsque cette variable y est travaillée de manière cohérente avec les deux autres variables.

\section{Étude qualitative : émotion et rationalité}

L'appui sur les résultats de l'expérience du couple émotion-rationalité permet de conjecturer certains éléments fondateurs de l'amélioration potentielle du ressenti des étudiants, ayant participé à cette expérience, à propos des mathématiques. Même si ces résultats ne sont pas l'objet de cet article, nous allons dans ce qui suit aborder succinctement ces éléments en focalisant sur deux des variables du questionnaire : les mathématiques en tant que science et l'apprentissage des mathématiques.

Le ressenti des mathématiques en tant que science concerne le caractère structuré des règles et des démarches qui fondent les mathématiques. Au fil des séances, les étudiants passent beaucoup plus facilement de la rationalité pragmatique à la rationalité théorique, les conflits de rationalité à ce sujet étant plus rapidement résolus (Lecorre, 2016). Les émotions vécues à ces moments, initialement très vives, éclats de voix, de rire, font place à des émotions davantage épistémiques et liées au doute, à la 
frustration de ne pas comprendre, à la joie de finalement comprendre (Lecorre 2018; Liljedahl et al., 2019).

Pour ce qui est du ressenti des mathématiques en tant que domaine d'apprentissage, on peut observer des changements de posture des étudiants : initialement en retrait, ils s'investissent, montrent de la curiosité, attendent et écoutent l'explication de leurs pairs. Les séances sont ponctuées de fous rires collectifs lorsque les arguments surprennent ou bien lorsqu'un étudiant montre avec vigueur à quel point il n'accepte pas de penser autrement que ce qu'il pense alors que les autres essaient de le convaincre. Ce vécu intense entre rationnel et émotionnel est une expérience qui déclenche du plaisir dans l'apprentissage et renforce le ressenti positif associé (Liljedahl, 2005).

\section{Limites de l'étude}

Malgré une cohérence certaine des résultats, un certain nombre de limites propres aux conditions de cette étude doivent être prises en compte afin d'appréhender la portée de ceux-ci.

L'étude d'un groupe témoin, sur un autre site de l'académie, ne bénéficiant pas du même cours magistral, aurait pu permettre de porter un avis plus tranché sur les effets de ce cours magistral sur le ressenti des étudiants. Initialement prévu comme une étude exploratoire, ce groupe témoin n'avait pas été envisagé.

La taille, relativement faible, de l'échantillon étudié (50), particulièrement lorsque celui-ci est réparti en groupes (A, B, C), constitue une limite significative de la présente étude.

L'étude porte sur les effets du cours magistral réalisé par l'un des auteurs de cet article. Aussi, malgré les précautions d'usage dans ce type de situation, on ne peut pas écarter la possibilité d'un biais méthodologique.

\section{Conclusion et perspectives}

Compte tenu des limites exprimées au paragraphe précédent, nous restons nuancés à propos des résultats. Un certain nombre d'indicateurs ont montré que cette expérimentation qui avait pour objectif d'améliorer le ressenti vis-à-vis des mathématiques à travers l'expérience des étudiants du couple (rationalité, émotion) dans la construction des notions mathématiques en cours magistral a plutôt conduit à des résultats conformes à l'objectif qui lui était assigné. Nous avons constaté également que la poursuite ou non en TD de ces objectifs par la même proposition pédagogique joue un rôle sur l'ampleur de ces changements de ressenti. Si ces deux constats conduisent à crédibiliser l'effet de l'expérience des étudiants dans ces cours sur leur ressenti vis-à-vis des mathématiques, il reste de nombreuses questions à étudier en utilisant les éléments recueillis lors de cette expérimentation ou en programmant de futures expérimentations.

Tout d'abord, pour cette expérimentation, une étude didactique et systématique des situations en matière de rationalité et d'émotion doit être réalisée à partir des vidéos réalisées lors de l'expérimentation. Peuton observer effectivement le jeu conjoint des rationalités et des émotions lors de la réalisation de ces cours magistraux? Lexpérience de ce couple (rationalité, émotion) à l'œuvre est-elle véritablement celle 
qui est attendue? Observe-t-on bien une progressive modification du sujet épistémique? Quels sont les effets sur la nature du doute, sur la persévérance dans l'action, sur la manière d'envisager les outils mathématiques? Des études ultérieures plus qualitatives, voire cliniques, pourraient être nécessaires pour répondre à ces questions.

Nous finirons cet article par la citation d'une étudiante sortant d'un examen de $4 \mathrm{~h}$ de mathématiques avec le sourire aux lèvres: "Avant je pleurais en allant en mathématiques, maintenant je joue en cours de mathématiques». Cette citation montre que le sujet de cette étude exploratoire mérite d'être poursuivi, pour tenter de répondre à la réelle détresse liée aux mathématiques de certains étudiants professeurs d'école. Elle montre aussi qu'une résilience est réalisable.

\section{Notes}

1 Création des ESPE (Établissement Supérieur du Professorat et de l'Enseignement) avec la loi Peillon 2013

2 Création des INSPE (Institut National Supérieur du Professorat et de l'Enseignement) avec la loi Blanquer 2019.

3 L'enquête internationale Pisa (2016) classe la France $26^{\mathrm{e}}$ sur 70 pays testés.

4 Rapport Cnesco (2016) : inégalité sociales et migratoires, comment l'école amplifie-t-elle les inégalités?

5 Le rapport TIMSS 2015 place la France au dernier rang des pays de l'OCDE pour les résultats moyens de fin de primaire en mathématiques.

\section{Références}

Adihou, A. (2011). Enseignement-apprentissage des mathématiques et souffrance à l'école. Les Collectifs du Cirp, $2,90-102$.

Amato, S-A. (2004). Improving student teachers' attitudes to mathematics. Dans Høines, M. J., Fuglestad, A. B., International Group for the Psychology of Mathematics Education, et Bergen University College (dir.), Proceedings of the 28th conference of the International Group for the Psychology of Mathematics Education (vol. 2, p. 25-32). Bergen University College Edition.

Arsenault, C. (2010). Le spectre de la maîtresse d'école : conceptions et résistances au développement des compétences professionnelles. Dans J. Proulx \& L. Gattuso (dir.), Formation des enseignants en mathématiques : tendances et perspectives actuelles (p. 121-125). Les Éditions du CRP.

Artigue, M. (1988). Ingénierie didactique. Recherche en Didactique des Mathématiques, 9(3), 281-308. La Pensée Sauvage.

Baldrighi, A., Bellinzona, C. et Pesci, A. (2011). Promoting resilience in students through cooperative learning experiences: a work in progress, Dans K. Kislenko (dir.), Current state of research on mathematical beliefs XVI Proceedings of the MAVI16 Conference (p. 7-27). Tallinn, Estonia Edited.

Baruk, S. (1973). Échec et Maths. Seuil.

Blanco, L., Guerrero, E., Caballero, A., Brígido, M. et Mellado, V. (2010). The affective dimension of learning and teaching mathematics and science. Dans M-P. Caltone (dir.), Handbook of Lifelong Learning Developments (p. 265-287). Nova Science Publishers.

Brousseau, G. (1998). Théorie des situations didactiques : didactique des mathématiques 1970-1990. La Pensée Sauvage.

Damasio A. (2005). Descartes' Error: Emotion, Reason, and the Human Brain. Penguin Edition

Hannula M., Liljedahl P., Kaasila R., \& Rösken B. (2007). Researching relief of mathematics anxiety among pre-service elementary school teachers. Dans Jeong-Ho Woo et al. (dir.), Proceedings of 31st Annual Conference for the Psychology of Mathematics Education, (vol. 1, p. 153-156). The Korea Society of Educational Studies in Mathematics. 
Lecorre T. (2016). Des conditions de conception d'une ingénierie relative à la défnition de limite- Un cadre basé sur un modèle de rationalité. [Thèse de doctorat, Université Grenoble-Alpes].

Lecorre T. (2018). Le bonheur par les mathématiques. Dans B. Mabilon-Bonfils et al. (dir.), Les écoles du bonheur. Pour une révolution éducative, (p. 25-41). Editions Teraedre.

Legrand, M. (1993). Débat scientifique en cours de mathématiques et spécificité de l'analyse. Repères IREM, 10, $123-158$.

Liljedahl, P. (2005). Mathematical discovery and affect: The effect of AHA! experiences on undergraduate mathematics students. International Journal of Mathematical Education in Science and Technology, 36(2-3), 219-234.

Liljedahl, P., Rösken, E., Rolka, K., (2019). Changes to preservice elementary teachers' beliefs about mathematics and the teaching and learning of mathematics: How and why? Journal of Adult Learning, Knowledge and Innovation, 1-11. https://doi.org/10.1556/2059.03.2019.09

Newton, K., Leonard, J., Evans, B., Eastburn, J., (2012). Preservice Elementary Teachers' Mathematics Content Knowledge and Teacher Efficacy. School Science and Mathematics, 112(5), 289-299.

Olson, A., Stoehr, K. (2019). From numbers to narratives: Preservice teachers experiences with mathematics anxiety and mathematics teaching anxiety. School Science and Mathematics, 119(2), 72-82.

Peix, A., Tisseron, C. (1998). Le problème ouvert comme moyen pour réconcilier les futurs professeurs d'école avec les mathématiques. Petit $x, 48,5-21$.

Pekrun, R., (2014). Emotions and Learning, educational practices. Bureau international d'éducation de l'UNESCO, Académie internationale d'éducation.

Ronzeau, M., Saint-Girons B. (2018). Quelles évolutions pour les concours de recrutement des enseignants? Ministère français de l'Éducation nationale. Paris.

Southwell B., Penglase M. (2005). Mathematical knowledge of pre-service primary teachers. Dans H.L. Chic,k et al. (dir.), Proceedings of the 29th Conference of the International Group for the Psychology of Mathematics Education, (vol. 4, 209-216). Melbourne PME edition.

Smith, C. (2010). Choosing more mathematics: happiness through work? Research in Mathematics Education, 12(2), 99-116.

Villani, C., Torrossian C., (2018). 21 mesures pour l'enseignement des mathématiques. Ministère français de l'Éducation nationale, Paris.

Vinson, B.M. (2001). A Comparison of Preservice Teachers'Mathematics Anxiety Before and After a Methods Class Emphasizing Manipulatives. Early Childhood Education Journal, 29, 89-94.

\section{Pour citer cet article}

Lecorre, T. et Ghedamsi-Lecorre, I. (2021). Étude exploratoire d'un changement potentiel de ressenti vis-à-vis des mathématiques de futurs professeurs des écoles en formation initiale. Formation et profession, 29(1), 1-14.

http://dx.doi.org/10.18162/fp.2021.581 